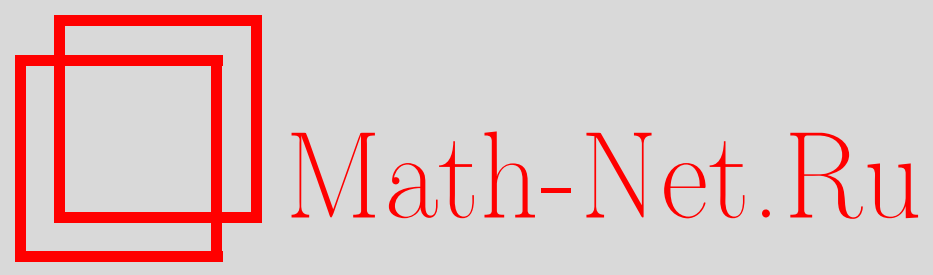

М. Б. Абросимов, Минимальные вершинные расширения направленных звезд, Дискрет. матем., 2011, том 23, выпуск 2, 93-102

DOI: https://doi.org/10.4213/dm1144

Использование Общероссийского математического портала Math-Net.Ru подразумевает, что вы прочитали и согласны с пользовательским соглашением http://www . mathnet.ru/rus/agreement

Параметры загрузки:

IP : 54.81 .137 .203

26 апреля 2023 г., $17: 56: 03$ 


\title{
Минимальные вершинные расширения направленных звезд
}

\author{
(ㄷ) 2011 г. М. Б. Абросимов
}

\begin{abstract}
Неориентированной звездой называется полный двудольный граф вида $K_{1, n}$. Направленной звездой называется направленный граф, симметризация которого является неориентированной звездой. В статье дается полное решение задачи описания всех минимальных вершинных $k$-расширений направленных звезд при любом натуральном $k$.
\end{abstract}

Ориентированным графом (далее орграфом) называется пара $\vec{G}=(V, \alpha)$, где $V$ - конечное непустое множество, называемое множеством вершин, а $\alpha$ - отношение на множестве $V$, называемое отношением смежности. Элементы множества $\alpha$ называются дугами. Дуга вида $(u, u) \in \alpha$ называется петлей. Орграф с универсальным отношением смежности называется полным и обозначается $\vec{K}_{n}$, где $n-$ число вершин. Граф с пустым отношением смежности называется вполне несвязным и обозначается $O_{n}$. Основные определения даются согласно [1].

Орграф с антисимметричным отношением смежности называется направленным графом или диграфом. Полный диграф без петель называется турниром. Транзитивный турнир - это турнир, у которого из существования дуг $(u, v)$ и $(v, w)$ вытекает существование дуги $(u, w)$.

Граф с симметричным и антирефлексивным отношением смежности называется неориентированным графом (далее графом). В неориентированном графе пара встречных дуг называется ребром и обозначается $\{u, v\}$.

Симметризацией орграфа $\vec{G}=(V, \alpha)$ называется граф $G=\left(V,\left(\alpha \cup \alpha^{-1}\right) \backslash \Delta\right)$, то есть симметризация орграфа получается заменой дуг ребрами и удалением петель.

Вложением графа $G_{1}=\left(V_{1}, \alpha_{1}\right)$ в граф $G_{2}=\left(V_{2}, \alpha_{2}\right)$ называется такое инъективное отображение $\varphi: V_{1} \rightarrow V_{2}$, что для всех $u, v \in V_{1}$ выполняется следующее условие: если $(u, v) \in \alpha_{1}$, то $(\varphi(u), \varphi(v)) \in \alpha_{2}$.

Два графа $G_{1}=\left(V_{1}, \alpha_{1}\right)$ и $G_{2}=\left(V_{2}, \alpha_{2}\right)$ называются изоморфными, если можно установить взаимно однозначное соответствие $\varphi: V_{1} \rightarrow V_{2}$, сохраняющее отношение смежности: $(u, v) \in \alpha_{1} \Longleftrightarrow(\varphi(u), \varphi(v)) \in \alpha_{2}$ для всех $u, v \in V_{1}$.

Изоморфизм графа на самого себя называется автоморфизмом. Две вершины графа называются подобными, если существует автоморфизм, при котором одна вершина является образом другой. Граф, все вершины которого попарно подобны, называется вершинносимметрическим.

Граф $G^{*}=\left(V^{*}, \alpha^{*}\right)$ называется минимальным вершинным $k$-расширением $n$-вершинного графа $G=(V, \alpha)$, если выполняются следующие условия: 
(1) граф $G^{*}$ является вершинным $k$-расширением $G$, то есть граф $G$ вкладывается в каждый подграф графа $G^{*}$, получающийся удалением любых его $k$ вершин;

(2) граф $G^{*}$ содержит $n+k$ вершин, то есть $\left|V^{*}\right|=|V|+k$;

(3) $\alpha^{*}$ имеет минимальную мощность при выполнении условий 1 и 2.

Граф $G^{*}$ называется точным вершинным $k$-расширением графа $G$, если любой граф, получающийся удалением произвольных $k$ вершин графа $G^{*}$, изоморфен графу $G$.

Граф $G_{t}=\left(V_{t}, \alpha_{t}\right)$ называется тривиальным $k$-расширением графа $G=(V, \alpha)$, если граф $G_{t}$ получается из графа $G$ добавлением $k$ вершин и соединением их со всеми вершинами графа $G$ и друг с другом.

Понятие минимального $k$-расширения введено на основе понятия оптимальной $k$-отказоустойчивой реализации, которое было предложено Хейзом в работе [2], где была описана модель для исследования отказоустойчивости, основанная на графах.

Технической системе $\Sigma$ сопоставляется помеченный граф $G(\Sigma)$, вершины которого соответствуют элементам системы $\Sigma$, ребра - связям между элементами, а метки указывают тип элементов. Под отказом элемента технической системы $\Sigma$ понимается удаление соответствующей ему вершины из графа системы $G(\Sigma)$ и всех связанных с ней ребер. Говорят, что система $\Sigma^{*}$ является $k$-отказоустойчивой реализацией системы $\Sigma$, если отказ любых $k$ элементов системы $\Sigma^{*}$ приводит к графу, в который можно вложить граф системы $\Sigma$ с учетом меток вершин. Построение $k$ отказоустойчивой реализации системы $\Sigma$ можно представить себе как введение в нее определенного числа новых элементов и связей. При этом предполагается, что в нормальном режиме работы избыточные элементы и связи маскируются, а в случае отказа происходит реконфигурация системы до исходной структуры.

Пусть в системе $\Sigma$ встречается $t$ различных типов элементов. Очевидно, что любая ее $k$-отказоустойчивая реализация должна содержать не менее $k$ дополнительных элементов каждого типа. Легко видеть, что такого числа дополнительных элементов достаточно для построения $k$-отказоустойчивой реализации системы $\Sigma$. В самом деле, добавим $k$ элементов каждого типа и соединим их все между собой и с элементами системы $\Sigma$. Тогда любой отказавший элемент можно будет заменить одним из добавленных элементов соответствующего типа. Построенную таким образом $k$-отказоустойчивую реализацию можно назвать тривиальной.

Назовем $k$-отказоустойчивую реализацию $\Sigma^{*}$ системы $\Sigma$, состоящей из $t$ элементов различного типа, оптимальной, если система $\Sigma^{*}$ отличается от системы $\Sigma$ на $k$ элементов каждого из $t$ типов системы $\Sigma$, и среди всех $k$-отказоустойчивых реализаций с тем же числом элементов система $\Sigma^{*}$ имеет наименьшее число ребер.

На практике, элементы технических систем часто оказываются однотипными. При исследовании отказоустойчивости в подобных системах метки элементов опускаются, и в качестве графа системы рассматривается граф без меток. В этом случае оптимальная $k$-отказоустойчивая реализация будет содержать в точности $k$ дополнительных элементов.

Хейз предложил процедуры построения оптимальной $k$-отказоустойчивой реализации для цепи, цикла и помеченного дерева. Позднее, Хейз совместно с Харари в [3] обобщили модель на случай отказов связей между элементами, предложив понятие реберной отказоустойчивости. Модель отказоустойчивости, в которой рассматриваются только отказы элементов, было предложено называть вершинной отказоустойчивостью (см. [4]). В той же работе было введено понятие точной $k$-отказоустойчивой реализации. В [5] удалось доказать, что рассматриваемые задачи являются вычислительно сложными: задачи распо- 
знавания вершинной или реберной $k$-отказоустойчивой реализации принадлежат классу NP-полных задач.

Граф называется двудольным, если множество его вершин $V$ может быть разбито на два подмножества вершин $V_{1}$ и $V_{2}$, таких, что концы любого ребра графа принадлежат разным подмножествам. Если граф содержит все ребра, соединяющие вершины из множеств $V_{1}$ и $V_{2}$, то граф называется полным двудольным графом и обозначается $K_{m, n}$, где $m$ и $n$ - число вершин во множествах $V_{1}$ и $V_{2}$.

Неориентированной звездой (далее просто звездой) или звездным графом называется полный двудольный граф вида $K_{1, n}$, где $n>0$ (то есть одна вершина смежна с $n$ несмежными вершинами). Ориентированной звездой будем называть орграф, симметризацией которого является звезда. Ориентированную звезду будем обозначать $Z_{m, n, p}$, где $m$ и $n-$ число вершин с единственной, соответственно, исходящей и входящей дугой, а $p$ - число вершин с одной входящей и одной исходящей дугой. Направленной звездой будем называть ориентированную звезду, являющуюся диграфом, и обозначать $Z_{m, n}$ (у направленной звезды $p=0)$. Вершину, являющуюся концом каждой дуги звезды, будем называть центральной.

В [6] удалось найти общее решение для описания минимальных вершинных $k$-расширений предполных графов, то есть графов, которые имеют хотя бы одну вершину, смежную со всеми остальными. Звездный граф является частным случаем предполного графа, и в [7] дается полное описание минимальных вершинных и реберных $k$-расширений для неориентированных звезд. Приведем соответствующие теоремы относительно вершинных $k$-расширений:

Теорема 1. Единственным минимальным вершинным 1-расширением звездного графа $K_{1, n}$ является тривиальное 1-расширение $K_{2, n}$.

Теорема 2. Для звездных графов $K_{1, n}$ справедливы следующие утверждения.

(1) При нечетном $n$ и любом натуральном $k$, звезда $K_{1, n}$ имеет единственное, с точностью до изоморфизма, минимальное вершинное $k$-расширение - тривиальное $k$-расширение.

(2) При четном $n$ :

(а) при четном $k$ число минимальных вершинных $k$-расширений звезды $K_{1, n}$ в точности равно числу ее неизоморфных минимальных вершинных $(k-1)$ расширений, причем каждое из минимальных вериинных k-расширений есть тривиальное 1-расширение соответствующего минимального вершинного $(k-1)$-расширения;

(b) при нечетном $k$ выделяются три случая:

- $k<n^{2}-4 n+1$ звезда $K_{1, n}$ имеет единственное, с точностью до изоморфизма, минимальное вершинное $k$-расширение - тривиальное $k$-расширение;

- при $k=n^{2}-4 n+1$ звезда $K_{1, n}$ имеет два, с точностью до изоморфизма, минимальных вершинньх $k$-расширения: тривиальное $k$-расширение $u$ граф $R_{n+k+1, n+k-1}$;

- при $k>n^{2}-4 n+1$ звезда $K_{1, n}$ имеет единственное минимальное вершинное $k$-расширение - граф $R_{n+k+1, n+k-1}$. 
Граф $R_{n, n-2}$ из формулировки теоремы - это однородный $n$-вершинный граф порядка $n-2$. В работе [6] доказывается, что такой граф имеет вид $O_{2}+\ldots+O_{2}$, где + обозначает операцию соединения графов: соединением двух графов $G_{1}=\left(V_{1}, \alpha_{1}\right)$ и $G_{2}=\left(V_{2}, \alpha_{2}\right)$, не имеющих общих вершин, называется граф

$$
G_{1}+G_{2}=\left(V_{1} \cup V_{2}, \alpha_{1} \cup \alpha_{2} \cup V_{1} \times V_{2} \cup V_{2} \times V_{1}\right) \text {. }
$$

Сформулируем и докажем лемму общего характера относительно связи минимальных вершинных $k$-расширений ориентированных и неориентированных графов.

Лемма 1. Пусть $\vec{G}^{*}$ - минимальное вериинное $k$-расширение орграфа $\vec{G}$. Тогда симметризация $\vec{G}^{*}$ является вериинным $k$-расширением симметризации $\vec{G}$.

Доказательство. Пусть $\vec{G}^{*}$ - минимальное вершинное $k$-расширение орграфа $\vec{G}$. Обозначим через $G$ симметризацию орграфа $\vec{G}$, а через $G^{*}-$ симметризацию орграфа $\vec{G}^{*}$. Выберем произвольный набор $F$, состоящий из $k$ вершин орграфа $\vec{G}^{*}$. По определению, орграф $\vec{G}^{*}-F$, который получается удалением из $\vec{G}^{*}$ всех вершин, входящих в $F$ вместе с дугами, допускает вложение орграфа $\vec{G}$. Это означает, что существует биекция $\varphi: V \rightarrow V^{*} \backslash F$, такая что

$$
\forall u, v \in V \quad((u, v) \in \alpha) \Longrightarrow\left((\varphi(u), \varphi(v)) \in \alpha^{*}\right) .
$$

По определению симметризации, наличие дуг $(u, v)$ и $(\varphi(u), \varphi(v))$ в орграфах $\vec{G}$ и $\vec{G}^{*}$ означает наличие ребер $\{u, v\}$ и $\{\varphi(u), \varphi(v)\}$ в соответствующих симметризациях $G$ и $G^{*}$, но тогда

$$
\forall u, v \in V \quad(\{u, v\} \in \beta) \Longrightarrow\left(\{\varphi(u), \varphi(v)\} \in \beta^{*}\right),
$$

то есть граф $G$ вкладывается в граф $G^{*}-F$.

Следствие 1. Число дополнительных дуг минимального вершинного $k$-расширения орграфа $\vec{G}$ не менее числа дополнительных ребер минимального вершинного $k$-расширения симметризациии орграфа $\vec{G}$.

Следствие 2. Пусть граф $G^{*}$ является минимальным вершинным $k$-расширением графа $G$, диграф $H$ есть некоторая ориентация графа $G$, а диграф $H^{*}$ есть некоторая ориентация графа $G^{*}$. Тогда, если $H^{*}$ является вериинным $k$-расширением диграфа $H$, то $H^{*}$ является и минимальным вершинным $k$-расширением диграфа $H$.

Предложение 1. Граф $Z T_{m, n, p}$, получающчйся из направленной звезды $Z_{m, n}$ добавлением $k=p-1$ копий иентральной вершинь и соединением их друг с другом по схеме транзитивного турнира, является вершинным $k$-расширением орграфа $Z_{m, n}$.

Доказательство. Рассмотрим граф $Z T_{m, n, p}$. Его вершины можно разделить на три группы.

Первая группа состоит из $p$ центральных вершин, соединенных по схеме транзитивного турнира.

Вторая группа состоит из $m$ вершин-источников, от каждой из которых исходят дуги ко всем вершинам первой группы. Очевидно, что все вершины этой группы подобны и имеют полустепени исхода и захода $(p, 0)$. 
Третья группа состоит из $m$ вершин-стоков, в каждую из которых заходят дуги от каждой вершины первой группы. Очевидно, что все вершины этой группы подобны и имеют полустепени исхода и захода $(0, p)$.

Рассмотрим орграф $\vec{G}$, получающийся из $Z T_{m, n, p}$ удалением $k$ произвольных вершин. Обозначим через $p_{1}, m_{1}$ и $n_{1}$ количество удаленных вершин первой, второй и третьей групп соответственно:

$$
\begin{gathered}
0 \leqslant p_{1} \leqslant p=k+1, \\
0 \leqslant m_{1} \leqslant m, \\
0 \leqslant n_{1} \leqslant n, \\
p_{1}+m_{1}+n_{1}=k .
\end{gathered}
$$

Покажем, что направленная звезда $Z_{m, n}$ вкладывается в орграф $\vec{G}$.

При $p_{1}=k$ (все удаленные вершины - вершины первой группы) орграф $\vec{G}$ изоморфен звезде $Z_{m, n}$ и, таким образом, допускает вложение.

Пусть $p_{1}<k$. В [8] было показано, что транзитивный турнир $T_{n+k}$ является точным вершинным $k$-расширением транзитивного турнира $T_{n}, n>1$. В нашем случае после удаления $p_{1}$ центральных вершин первой группы оставшиеся $p-p_{1}>1$ вершины будут по-прежнему образовывать транзитивный турнир $T_{p-p_{1}}$. При этом

$$
p-p_{1}=k+1-p_{1}=m_{1}+n_{1}+1 \text {. }
$$

Вершины транзитивного турнира, как известно, могут быть упорядочены по отношению достижимости. Обозначим оставшиеся центральные вершины в соответствующем порядке через $u_{1}, \ldots, u_{m_{1}+n_{1}+1}$. Таким образом, из любой вершины с меньшим индексом идет дуга в любую вершину с большим индексом.

Укажем соответствие вершин звезды $Z_{m, n}$ вершинам орграфа $\vec{G}$, которое определяет вложение:

(a) $m$ вершинам-источникам соответствуют $m-m_{1}$ оставшихся вершин второй группы и $m_{1}$ вершин $u_{1}, \ldots, u_{m_{1}}$;

(б) $n$ вершинам-стокам соответствуют $n-n_{1}$ оставшихся вершин третьей группы и $n_{1}$ вершин $u_{m_{1}+2}, \ldots, u_{m_{1}+n_{1}+1}$;

(в) вершина $u_{m_{1}+1}$ соответствует центральной вершине звезды $Z_{m, n}$.

Видно, что из каждой вершины типа (а) идет дуга в вершину $u_{m_{1}+1}$, а из вершины $u_{m_{1}+1}$ идет дуга в каждую вершину типа (б); таким образом, звезда $Z_{m, n}$ действительно вкладывается в орграф $\vec{G}$.

Из леммы 1, предложения 1 и теоремы 1 получаем следующее утверждение.

Теорема 3. Пусть $Z_{m, n}$ направленная звезда, причем $m+n>1 u m n \neq 1$. Диграф $Z T_{m, n, 2}$, получающийся из направленной звездьl $Z_{m, n}$ добавлением копии ичентральной вершины и соединением их друг с другом дугой, является единственным, с точностью до изоморфизма, минимальным вериинным 1-расширением орграфа $Z_{m, n}$.

Замечание 1. Направленная звезда $Z_{1,1}$ является направленной цепью $P_{3}$, и ее единственное минимальное вершинное 1-расширение, являющееся также и ее точным вершинным 1-расширением, есть направленный цикл $C_{4}$. 


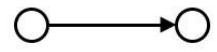

a)

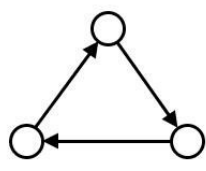

б)

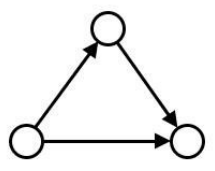

b)

Рис. 1. Звезда $Z_{1,0}$ и два ее минимальных вершинных 1-расширения

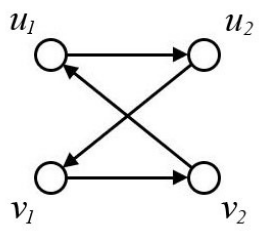

Рис. 2. Схема ориентации ребер

Замечание 2. Направленная звезда $Z_{1,0}$ изоморфна звезде $Z_{0,1}$ и имеет два неизоморфных минимальных вершинных 1-расширения, каждое из которых является и точным вершинным 1-расширением, именно, транзитивную и циклическую тройки (см. рис. 1).

Из леммы 1, предложения 1 и теоремы 2 получаем следующее утверждение.

Теорема 4. Диграф $Z T_{m, n, p}$, получающийся из направленной звезды $Z_{m, n}$ добавлением $k=p-1$ копий иентральной вершины и соединением их друг с другом по схеме транзитивного турнира, является единственньм, с точностью до изоморфизма, минимальньмм вериинныл $k$-расширением орграфа $Z_{m, n}$, если выполняется любое из двух условий:

(1) $m+n$ нечетно;

(2) $k<n^{2}-4 n+1$.

Далее необходимо рассмотреть случай, когда $m+n$ четно, так как в этом случае, согласно теореме 2, могут появиться вершинные $k$-расширения с меньшим числом дополнительных дуг.

Рассмотрим схему ориентации однородного $(2 n)$-вершинного графа $R_{2 n, 2 n-2}$. Напомним, что граф $R_{2 n, 2 n-2}$ может быть представлен в виде $O_{2}+\ldots+O_{2}$. Будем ориентировать ребра от каждой пары вершин из $O_{2}$ до каждой другой такой пары. От первой вершины пары направим одну исходящую дугу в первую вершину второй пары и входящую дугу от второй вершины второй пары, а из второй вершины первой пары направим дуги в обратном порядке, так что получится ориентированный цикл. Если $u_{1}$ и $v_{1}-$ вершины первой пары, а $u_{2}$ и $v_{2}-$ вершины второй пары, то ориентируем дуги следующим образом: $\left(u_{1}, u_{2}\right),\left(v_{1}, v_{2}\right),\left(v_{2}, u_{1}\right),\left(u_{2}, v_{1}\right)$ (см. рис. 2$)$. Продолжаем ориентацию описанным способом по всем парам вершин. Обозначим построенный диграф через $D_{2 n, 2 n-2}$.

Отметим некоторые достаточно очевидные свойства диграфа $D_{2 n, 2 n-2}$.

1. Отображение $u_{i} \rightarrow v_{i}, v_{i} \rightarrow u_{i}, i=1, \ldots, n$, является автоморфизмом. 


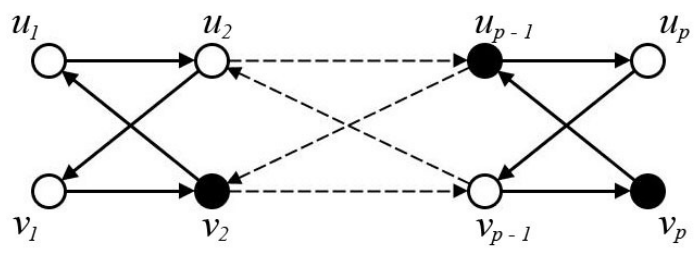

Рис. 3. Диграф $D_{2 p, 2 p-2}$

2. Отображение $u_{i} \rightarrow u_{i+1}, v_{i} \rightarrow v_{i+1}, i=1, \ldots, n-1, u_{n} \rightarrow v_{1}, v_{n} \rightarrow u_{1}$ является автоморфизмом.

3. Все вершины диграфа $D_{2 n, 2 n-2}$ подобны и имеют полустепени исхода и захода, равные $n-1$ (следует из свойств 1 и 2).

4. Диграф $D_{2 n, 2 n-2}$ является вершинно-симметрическим графом (следует из свойства 3 ).

5. При удалении любой пары несмежных вершин $u_{i}$ и $v_{i}$ диграфа $D_{2 n, 2 n-2}$ получается диграф $D_{2 n-2,2 n-4}$.

6. Все максимальные подграфы диграфа $D_{2 n, 2 n-2}$ изоморфны. Обозначим граф, получающийся удалением любой вершины диграфа $D_{2 n, 2 n-2}$, через $T D_{2 n-2,2 n-4}$.

Предложение 2. Диграф $D_{2 n+k+1,2 n+k-1}$ при нечетном $k$ является вершинным $k$-расширением направленной звезды $Z_{n, n}$.

Доказательство. Обозначим число вершин диграфа $D_{2 n+k+1,2 n+k-1}$ через $2 p$ : $2 p=2 n+k+1$. Как и ранее, вершины диграфа $D_{2 n+k+1,2 n+k-1}$ обозначим через $u_{i}$ и $v_{i}, i=1, \ldots, p$.

Рассмотрим орграф, получающийся из $D_{2 n+k+1,2 n+k-1}$ удалением произвольных $k$ вершин: $u_{i_{1}}, \ldots, u_{i_{k_{1}}}$ и $v_{i_{1}}, \ldots, v_{i_{k_{2}}}: k_{1}+k_{2}=k$. Обозначим этот орграф через $\vec{G}$. Так как $k$ нечетное, то $k_{1} \neq k 2$. Пусть, для определенности, $0 \leqslant k_{1}<k_{2} \leqslant k$. На рис. 3 для наглядности оставлена только часть дуг: дугами соединяются вершины одной горизонтали от вершины с меньшим индексом к вершине с большим и вершины разных горизонталей, от вершины с большим индексом к вершине с меньшим. Удаленные вершины на рисунке помечены черным (при $p=4$ этот рисунок соответствовал бы случаю поиска вершинного 3-расширения для 5-вершинной звезды $Z_{2,2}$ ). Для краткости будем называть удаленные вершины черными, а оставшиеся - белыми. Количество белых и черных вершин, очевидно, равно $2 n+1$ и $k$ штук соответственно.

Чтобы доказать, что диграф $D_{2 n+k+1,2 n+k-1}$ является вершинным $k$-расширением звезды $Z_{n, n}$, достаточно показать, что в орграфе $\vec{G}$ есть вершина с $n$ входящими и $n$ исходящими дугами. Кандидатами для такой вершины могут быть только такие вершины, у которых парная была удалена, так как только они соединены дугами со всеми остальными вершинами. Искомых вершин может быть и несколько. Для каждой вершины $w$ введем в рассмотрение индекс, состоящий из пары чисел $d^{+}(w)$ и $d^{-}(w)$ :

$d^{-}(w)$ есть число белых вершин слева от $w$ в ее ряду плюс число белых вершин справа от $w$ в другом ряду; 


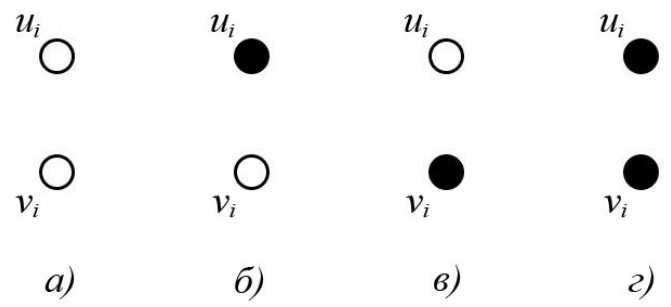

Рис. 4. Пары вершин

Таблица 1. Изменение индексов

\begin{tabular}{|c|c|c|c|}
\hline Левая пара & Правая пара & $d^{+}$ & $d^{-}$ \\
\hline (б) & (б) & -1 & +1 \\
\hline (б) & (в) & 0 & 0 \\
\hline (в) & (б) & +1 & -1 \\
\hline (в) & (в) & +1 & -1 \\
\hline
\end{tabular}

$d^{+}(w)$ есть число белых вершин справа от нее в ее ряду плюс число белых вершин слева от нее в другом ряду.

По построению видно, что для вершин одной пары выполняются соотношения $d^{+}\left(u_{i}\right)=d^{-}\left(v_{i}\right)$ и $d^{-}\left(u_{i}\right)=d^{+}\left(v_{i}\right)$. Значение индексов $d^{+}(w)$ и $d^{-}(w)$ для белой вершины, соответственно, равны полустепеням исхода и захода. Искомая вершина, очевидно, должна удовлетворять условию $d^{+}(w)=d^{-}(w)=n$.

Покажем, что такая вершина найдется. На рис. 4 представлены четыре возможные конфигурации парных вершин.

Посчитаем суммы индексов для вершин из разных пар.

Для пары типа (a) $d^{+}(w)+d^{-}(w)=2 n-1$ (обе белые вершины пары не участвуют в подсчете индексов).

Для пар типа (б) и (в) $d^{+}(w)+d^{-}(w)=2 n$ (единственная белая вершина пары не участвует в подсчете индексов).

Для пары типа (г) $d^{+}(w)+d^{-}(w)=2 n+1$ (все белые вершины участвуют в подсчете индексов).

Для удобства будем считать, что пары типа (г), если они есть, располагаются справа, а пары типа (а), если они есть, по свойству 5 диграфа можно расположить слева, таким образом, представляющие для нас интерес пары типа (б) и (в) располагаются между парами типа (а) и (г). Обозначим для определенности номер первой пары интересующего нас интервала через $l$, а номер последней пары - через $r$.

Для каждой вершины пар (б) и (в) рассчитаем индекс. Вершина $u_{l}$ первой пары получит индекс $\left(n-k_{2}, n-k_{1}-1\right)$, если пара имеет тип (в), или $\left(n-k_{2}-1, n-k_{1}\right)$, если пара имеет тип (б). Вершина $u_{r}$ последней пары получит индекс $\left(n-k_{1}, n-k_{2}-1\right)$, если пара имеет тип (б), или $\left(n-k_{1}-1, n-k_{2}\right)$, если пара имеет тип (в). Так как $k_{1}<k_{2}$, то у вершины первой пары $d^{+}\left(u_{l}\right) \leqslant d^{-}\left(u_{l}\right)$, а у последней пары $d^{+}\left(u_{r}\right) \geqslant d^{-}\left(u_{r}\right)$. Изменение индексов при переходе от одной пары к другой при просмотре слева направо показано в табл. 1.

Таким образом, при переходе от одной пары к другой компоненты индекса либо не 


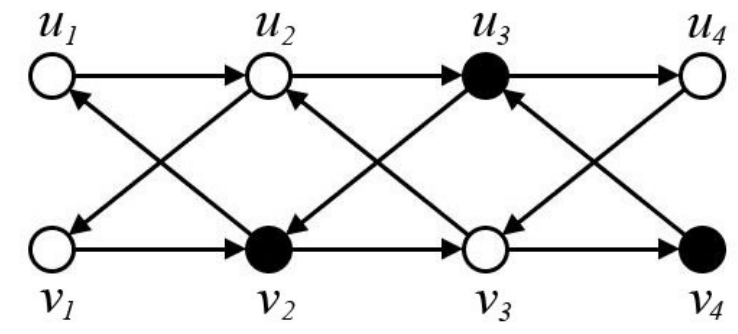

Рис. 5. Диграф $D_{8,6}$

изменяются, либо изменяются на 1. У первой пары $d^{+}\left(u_{l}\right) \leqslant d^{-}\left(u_{l}\right)$, а у последней пары наоборот: $d^{+}\left(u_{r}\right) \geqslant d^{-}\left(u_{r}\right)$. Следовательно, существует пара, у которой компоненты окажутся равными. Белая вершина из такой пары и будет искомой вершиной.

Пример 1. На рис. 5 представлена схема, иллюстрирующая доказательство - поиск вложения звезды $Z_{2,2}$ в ее 3-расширение. Рассчитаем индексы для верхнего ряда вершин: $u_{1}:(2,1), u_{2}:(2,2), u_{3}:(2,2), u_{4}:(2,2)$.

Таким образом, любая из вершин $u_{2}, v_{3}$ или $u_{4}$ является искомым образом для центральной вершины звезды.

Предложение 3. Диграф $T D_{2 n+k, 2 n+k-2}$ при четном $k$ является вершинным $k$-расширением направленной звезды $Z_{n, n}$.

Это предложение следует из предыдущего предложения, свойства 6 и очевидного замечания, что максимальный подграф вершинного $k$-расширения некоторого графа является для него вершинным $(k-1)$-расширением.

Предложение 4. Пусть натуральнье $m, n, k$ таковы, что $m \neq n, m+n$ четно, a $k$ нечетно. Никакой диграф, получающийся ориентачией однородного $(m+n+k+1)$ вершинного графа $R_{m+n+k+1, m+n+k-1}$, не является вершинныл $k$-расширением ни для какой направленной звезды $Z_{m, n}$.

Для доказательства достаточно показать, что предложение не выполняется уже при $k=1$. Предположим противное: пусть орграф $\vec{G}-$ некоторая ориентация графа $R_{m+n+2, m+n}-$ является вершинным $k$-расширением для направленной звезды $Z_{m, n}$, причем $m \neq n$ и $m+n$ четно. Не ограничивая общности, будем считать, что $m>n$ (так как $m+n$ четно, то справедливо даже $m>n+1)$. При удалении любой вершины $v$ графа $R_{m+n+2, m+n+k}$ получается граф вида $O_{1}+O_{2}+\ldots+O_{2}$, в котором есть только одна полная вершина $u$ - та, которая была несмежна с удаленной вершиной $v$. Следовательно, при удалении любой вершины $v$ орграфа $\vec{G}$ соответствующая вершина $u$ должна иметь полустепени захода и исхода не меньше, чем $m$ и $n$ соответственно. В силу произвольности выбора $v$ получаем, что любая вершина орграфа $\vec{G}$ должна иметь полустепени захода и исхода не меньше, чем $m$ и $n$ соответственно. Но тогда в каждую вершину орграфа $\vec{G}$ должно заходить не менее $m$ дуг, откуда получаем, что общее число дуг будет не менее $m(m+n+2)$. Однако количество ребер графа $R_{m+n+2, m+n}$ есть $(m+n+2)(m+n) / 2<(m+n+2)(m+m) / 2=m(m+n+2)$. Полученное противоречие доказывает предложение. 
С учетом предложений 2, 3 и 4, можно уточнить теорему 4.

Теорема 5. Диграф $Z T_{m, n, p}$, получающийся из направленной звезды $Z_{m, n}$ добавлением $k=p-1$ копий иеетральной вершины и соединением их друг с другом по схеме транзитивного турнира, является при $m \neq n$ единственным, с точностью до изоморфизма, минимальным вершинным $k$-расширением орграфа $Z_{m, n}$.

Теорема 6. Диграф $T D_{2 n+k, 2 n+k-2}$ при четном $k$ или максимальный подграф диграфа $T D_{2 n+k+2,2 n+k}$ при нечетном $k$ являются единственными минимальными вершинными $k$-расширениями направленной звезды $Z_{n, n}$.

\section{Список литературы}

1. Богомолов А. М., Салий В. Н., Алгебраические основы теории дискретных систем. Наука, Москва, 1997.

2. Hayes J. P., A graph model for fault-tolerant computing system. IEEE Trans. Comput. (1976) C25, №9, 875-884.

3. Harary F., Hayes J. P., Edge fault tolerance in graphs. Networks (1993) 23, 135-142.

4. Harary F., Hayes J. P., Node fault tolerance in graphs. Networks (1996) 27, 19-23.

5. Абросимов М. Б., О сложности некоторых задач, связанных с расширениями графов. Математические заметки (2010) 88, №5, 643-650.

6. Абросимов М. Б., Минимальные $k$-расширения предполных графов. Известия ВУЗов: Математика (2003) 493, №6, 3-11.

7. Абросимов М. Б., Минимальные расширения неориентированных звезд. В сб.: Теоретические проблемы информатики и ее приложений, 7. СГУ, Саратов, 2006, с. 3-5.

8. Абросимов М. Б., Минимальные расширения транзитивных турниров. Вестник ТГУ (2006), № 17 187-190.

Статья поступила 15.10.2008. 